\title{
Water-Wise Cities, ¿puede el patrimonio cultural del agua aportar estrategias ante el futuro cambio hidrológico?
}

Celia López-Bravo, José Peral López | Dpto. de Historia, Teoría y Composición Arquitectónicas, Universidad de Sevilla

URL de la contribución <www.iaph.es/revistaph/index.php/revistaph/article/view/4963>

Nos encontramos en un momento en el que el agua es totalmente dependiente de modelos tecnológicos de consumo energético, recursos financieros y un mercado privado altamente competitivo (Marat-Mendes, Mourão y d'Almeida 2016, 159) y, pese a que las ciudades europeas disponen de avanzados sistemas de captación, depuración, distribución y recogida de aguas, se prevé que el proceso de hiperurbanización lleve a 2,5 mil millones de nuevos habitantes a las ciudades (ONU 2018a). Además de este desplazamiento demográfico, nuestro planeta se avecina a un profundo cambio hidrológico, de modo que, actualmente, la gestión de los recursos y las infraestructuras constituyen el principal desafío de la ciudad del futuro.

Ante este complicado escenario, han sido puestas en marcha múltiples iniciativas de gobernanza por parte de diversas entidades y organizaciones a nivel mundial, entre las que destacan el establecimiento del periodo 2018-2028 como el Decenio Internacional para la Acción "Agua para el Desarrollo Sostenible" por parte de Naciones Unidas (ONU 2018b) o la elaboración de la guía 17 Principios para las ciudades water-wise elaborada por la International Water Association (IWA 2017a).

Estos principios se sustentan en 4 niveles de acción entre los que cabe destacar: la implementación de un diseño urbano sensible al agua, la mayor relación de la ciudad con su cuenca hidrológica y la creación de comunidades water-wise. Para ello entran en juego la planificación de diseños urbanos que propicien servicios regenerativos de agua, la acción urbana en la gestión de las cuencas y el aumento de la visibilidad del agua a través del diseño de infraestructuras verdes y azules que ofrezcan oportunidades de inclusión social (IWA 2017b).
Para alcanzar todas estas estrategias, que apuntan al (re)conocimiento del entorno hidrográfico y al cuestionamiento social sobre cómo habitamos las ciudades y qué uso hacemos de los recursos, la valoración del patrimonio cultural del agua y de determinadas infraestructuras históricas resulta el primer paso a considerar por los municipios. El estudio de estos elementos intrínsecos de la forma urbana nos permite comprender, además de su innegable valor histórico y técnico, la estructura y el funcionamiento ambiental de las ciudades en el pasado. Esta base histórica puede suponer importantes claves a la hora de reducir la contaminación y el impacto ambiental de los sistemas contemporáneos, acortar las cadenas de suministro o restablecer relaciones territoriales.

La evolución del abastecimiento de agua en las ciudades hasta alcanzar los sistemas sanitarios modernos es discontinua y variada en función de los condicionantes técnicos, económicos, políticos y, fundamentalmente, geográficos. Los primeros sistemas griegos, basados en la complementariedad de depósitos privados y redes públicas, y el perfeccionamiento, la centralización y la monumentalidad de los sistemas romanos constituyen una primera época de esplendor, en la que afloran necesidades como la seguridad del agua, la materialidad o el mantenimiento de las infraestructuras. Menos reconocida es, en este aspecto, la Edad Media, una época oscura en la que las ciudades no alcanzaron los equipamientos ni el esplendor clásico, marcada por la inestabilidad política y económica y las limitaciones tecnológicas. Es, en cambio, un periodo de interés para el estudio de la gestión del agua. En esta época, se desarrolla una cultura del agua basada en el uso racional, la seguridad y el aprovechamiento máximos, el mantenimiento de las instalaciones y la reutilización. Son pocos los ejemplos de ciudades medievales que emprendieron 
a debate Patrimonio cultural y cambio climático

| coordina Alejandro García Hermida

sofisticados sistemas de abastecimiento, pero casi todos los de este reducido grupo perpetuaron su uso hasta inicios del siglo $\mathrm{XX}$, desarrollando comunidades prósperas y competitivas.

En Europa, algunas ciudades italianas como Venecia, Siena o Bolonia constituyen ejemplos de gran interés. El aprovechamiento de sus particulares condiciones geográficas -sobre el agua, en el caso de Venecia; en altura, en el caso de Siena; o en piedemonte, en el caso boloñés- las llevaron a adelantarse a los preceptos actuales de conectividad ecológica.

Sus proyectos urbanos contaban con un enfoque sistémico, en el que reciclaban estructuras existentes y consideraban un todo al conjunto del sistema hídrico y el espacio público urbano. Desde las cisternas lagunares en los campi vencianos (Rizzi 2020), las fuentes públicas en ladera y el sistema de bottini sienés (Constantini y Martini 2010) y el imbricado sistema de canales y la llamada chiavica boloñesa (Lucci y Ruzzon 2013), estas ciudades superaban las estrategias coetáneas más comunes en la materia, como la recogida doméstica y las captaciones superficiales de agua a nivel privado. Introduciendo el recurso y la necesidad del agua en el
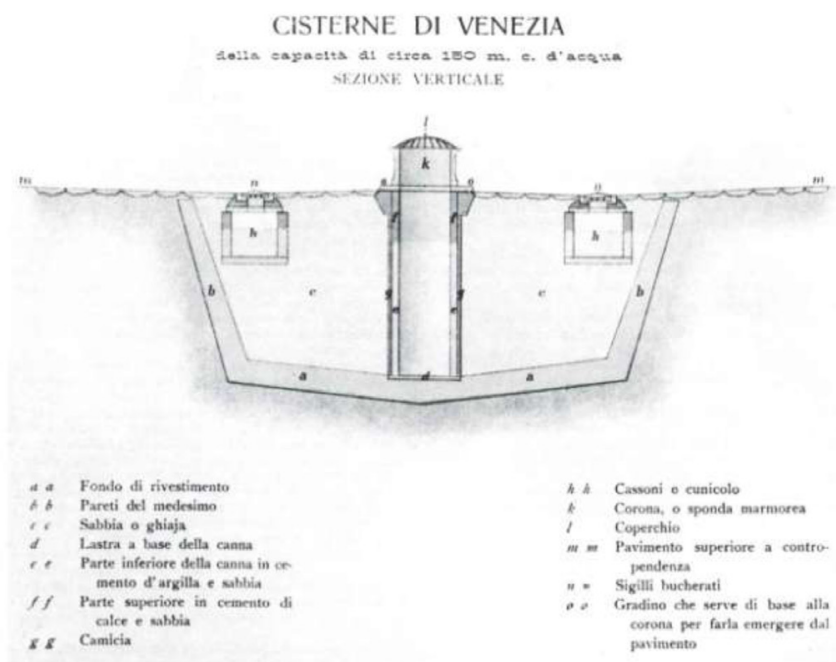

Sección de un pozo veneciano y su área de dotación | fuente Bianco 2020

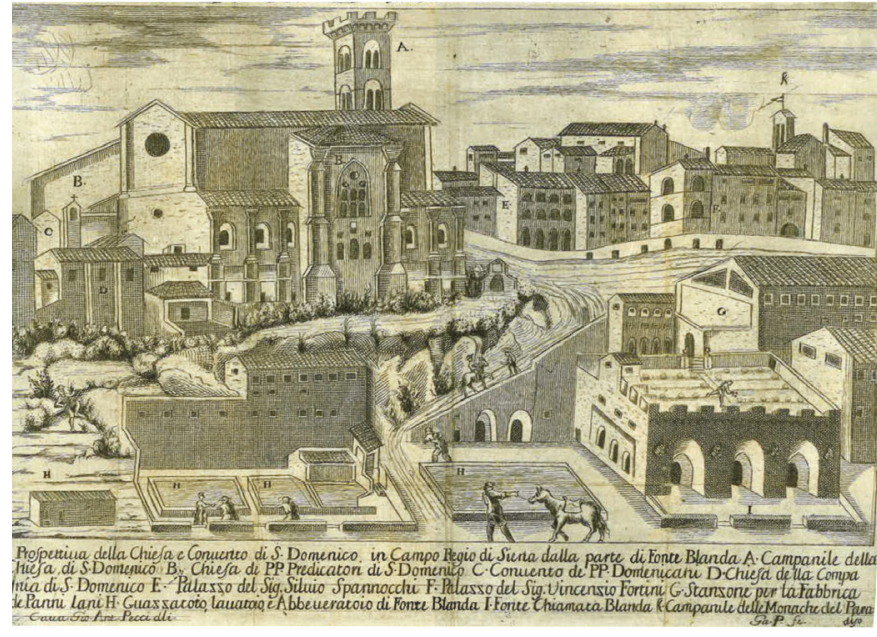

Dibujo de Fonte Branda en el que se representan los edificios principales y las funciones de cada una de las superficies de agua | fuente Pecci G.A. Ristretto delle cose più notabili della città di Siena ad uso de' forestieri ricorretto, e accresciuto. Siena, Bonetti nella stamp. Del pubblico per Francesco Rossi stampatore, 1759

diseño de sus edificios y espacios públicos, aportaron gran innovación y complejidad a su trazado, empleando el agua para consumo humano y como motor de una primitiva industrialización.

Máximas como el aprovechamiento de agua de precipitación, la autosuficiencia y el empleo de recursos "kilómetro 0", el mantenimiento y la conciencia ciudadana del cuidado, el uso racional del agua -cuyo derroche llegaba a ser considerado un delito mayor-y su reutilización, son hoy considerados principios de las llamadas waterwise cities que ya estaban presentes en estas infraestructuras preindustriales.

Evidentemente, hoy estos criterios se enmarcan en un contexto tecnológico distinto, donde los estándares de caudal de uso, calidad y salubridad de agua son otros. Pero su estudio puede colaborar en el diseño de estrategias más conscientes, apoyadas en valores y técnicas tradicionales de cara al futuro. Además, su reutilización constituye una gran oportunidad para suplementar el ciclo integral del agua urbana, reduciendo el consumo energético y el daño ambiental derivado de ciertos procesos de potabilización como la desalinización. 


\section{BIBLIOGRAFÍA}

- Bianco, G. "Relazione tecnica del 1860", Comune di Venezia. En: Rizzi, A. (2020) Vere da pozzo di Venezia. Caselle di Sommacampagna (Verona): Cierre Edizioni (Quaderni delle Regaste, 14)

- Costantini, A. y Martini, P. (2010) Landscape influence on the development of the medieval City-State of Siena, Italy. Italian Journal of Quaternary Sciences, n. ${ }^{\circ} 23$, pp. 283-298

- International Water Association [IWA] (2017a) The 17 IWA Principles for Water-Wise Cities. Disponible en: https:// iwa-network.org/projects/water-wise-cities/\#download_the principles [Consulta: 07/06/2021]

- International Water Association [IWA] (2017b) Los principios de la IWA para las ciudades Water-Wise. Disponible en: https:// iwa-network.org/wp-content/uploads/2017/06/IWA Brochure Water_Wise_Communities_screen_SP.pdf - [Consulta 07/06/2021]

- Lucci, P. y Ruzzon, D. (2013) Citta' d'acqua: Matera, Bologna e Modena. En: Focus su Acque e ambiente urbano. Qualità dell'ambiente urbano IX Rapporto. Roma: Istituto Superiore per la Protezione e la Ricerca Ambientale, pp. 41-51

- Marat-Mendes, T., Mourão, J. y Almeida, P.B. (De) (2016) Access to water in the Lisbon region in 1900. Water History, vol. 8, n. ${ }^{\circ}$ 2, pp. 159-189. Disponible en: https://doi.org/10.1007/ s12685-016-0160-3 [Consulta 14/06/2021]

- ONU [Organización de las Naciones Unidas] (2018a) Las ciudades seguirán creciendo sobre todo en los países en desarrollo, 16 de mayo de 2018. Disponible en: https://www. un.org/development/desa/es/news/population/2018-worldurbanization-prospects.html [Consulta: 09/06/2021]

- ONU [Organización de las Naciones Unidas] (2018b) Decenio Internacional para la Acción "Agua para el Desarrollo Sostenible". Disponible en: https://www.un.org/es/events/ waterdecade/ [Consulta 09/06/2021]izzi, A. (2020) Vere da pozzo di Venezia. Verona: Cierre Edizioni 